\title{
Erratum: Single-cell dynamics of the chromosome replication and cell division cycles in mycobacteria
}

Isabella Santi, Neeraj Dhar, Djenet Bousbaine, Yuichi Wakamoto \& John D. McKinney

Nature Communications 4:2470 doi:10.1038/ncomms3470 (2013); Published 16 Sep 2013; Updated 3 Dec 2013

There were errors associated with the Supplementary Movies in the version of this Article originally published. The legend of Supplementary Movie 5 was incorrectly associated with Supplementary Movie 4 and Supplementary Movie 5 was omitted. This has now been corrected in the HTML version of the Article; the PDF was correct from the time of publication. 